\title{
Fine structure in the beta-delayed proton decay of ${ }^{33} \mathrm{Ar}$
}

\author{
A. Honkanen ${ }^{1}$, L. Axelsson ${ }^{2}$, J. Äystö ${ }^{1,3}$, M.J.G. Borge ${ }^{4}$, B. Jonson ${ }^{2}$, \\ A. Jokinen ${ }^{3}$, I. Martel ${ }^{4}$, G. Martinez-Pinedo ${ }^{5}$, I. Mukha ${ }^{6}$, T. Nilsson ${ }^{2}$, G. Nyman ${ }^{2}$, \\ B. Petersen ${ }^{5}$, A. Poves ${ }^{6}$, M.H. Smedberg ${ }^{2}$, A. Teijeiro ${ }^{4}$, O. Tengblad ${ }^{3,4}$ \\ and the ISOLDE Collaboration
}

1) Department of Physics, University of Jyväskylä, FIN-40351 Jyväskylä, Finland

2) Fysiska Institutionen, Chalmers Tekniska Högskola, S-41296 Göteborg, Sweden

3) PPE Division, CERN, CH-1211 Genève 23, Switzerland

4) Instituto de Estructura de la Materia, CSIC, E-28006 Madrid, Spain

5) Departemento de Fisica Teorica, Universidad Autonoma de Madrid, Cantoblanco, E-28049 Madrid, Spain

6) Institut for Fysik og Astronomi, Aarhus Universitet, DK-8000 Aarhus C, Denmark

\begin{abstract}
Low energy beta-delayed protons from ${ }^{33} \mathrm{Ar}$ have been measured for the first time. The data reveal states, which, despite unfavourable barrier penetrability values, strongly decay to the first excited $2^{+}$state in ${ }^{32} \mathrm{~S}$. The observation is discussed in terms of the standard shell model. A natural explanation is provided by the large spectroscopic amplitudes, involving $\mathrm{s}_{1 / 2}$ and $\mathrm{d}_{3 / 2}$ orbitals, as well as the $l=0$ barrier penetrability, favouring the decay to the $2^{+}$state.
\end{abstract}

(IS339)

(Accepted for publication in Nucl. Phys. A)

RADIOACTIVITY: ${ }^{33} \mathrm{Ar}$ (from $1 \mathrm{GeV}$ p on CaO-target, selective mass separation); measured beta-delayed protons, $\mathrm{E}_{\mathrm{p}}, \mathrm{I}_{\mathrm{p}}$; deduced relative spectroscopic amplitudes; shell model calculation. Gas-Si telescope spectrometer. 



\title{
Fine structure in the beta-delayed proton decay of ${ }^{33} \mathrm{Ar}$
}

\author{
A. Honkanen ${ }^{1}$, L. Axelsson ${ }^{2}$, J. Äystö ${ }^{1,3}$, M.J.G. Borge ${ }^{4}$, B. Jonson ${ }^{2}$, \\ A. Jokinen ${ }^{3}$, I. Martel ${ }^{4}$, G. Martínez-Pinedo ${ }^{5}$, I. Mukha ${ }^{6}$, T. Nilsson ${ }^{2}$, G. Nyman ${ }^{2}$, \\ B. Petersen ${ }^{6}$, A. Poves ${ }^{5}$, M.H. Smedberg ${ }^{2}$, A. Teijeiro ${ }^{4}$, O. Tengblad ${ }^{3,4}$ \\ and the ISOLDE Collaboration
}

January 6, 1997

1. Department of Physics, University of Jyväskylä, FIN-40351 Jyväskylä, Finland

2. Fysiska Institutionen, Chalmers Tekniska Högskola, S-412 96 Göteborg, Sweden

3. PPE Division, CERN, CH-1211 Genève 23, Switzerland

4. Instituto de Estructura de la Materia, CSIC, E-28006 Madrid, Spain

5. Departemento de Fisica Teorica, Universidad Autonoma de Madrid, Cantoblanco, E-28049 Madrid, Spain

6. Institut for Fysik og Astronomi, Aarhus Universitet, DK-8000 Aarhus C, Denmark

\begin{abstract}
Low energy beta-delayed protons from ${ }^{33}$ Ar have been measured for the first time. The data reveal states, which, despite unfavourable barrier penetrability values, strongly decay to the first excited $2^{+}$state in ${ }^{32} \mathrm{~S}$. The observation is discussed in terms of the standard shell model. A natural explanation is provided by the large spectroscopic amplitudes, involving $s_{1 / 2}$ and $d_{3 / 2}$ orbitals, as well as the $l=0$ barrier penetrability, favouring the decay to the $2^{+}$state.
\end{abstract}

RADIOACTIVITY ${ }^{33} \mathrm{Ar}$ (from $1 \mathrm{GeV}$ p on Nb-foil target, selective mass separation); measured beta-delayed protons, $E_{p}, I_{p}$; deduced relative spectroscopic amplitudes; shell model calculation. Gas-Si telescope spectrometer. 


\section{Introduction}

Beta-delayed charged particle decay is a dominant decay mode for proton-rich nuclei. It provides high sensitivity and resolution in probing the properties of high-lying excited states and beta decay properties of nuclei with large proton excess. However, due to experimental limitations, such decays have not been well-studied for charged particle energies below $1 \mathrm{MeV}$. These low energy particles are expected to be present because of the favourable phase space of beta decay and the feeding of the excited states in the final nucleus. The low energy regime is of particular interest for the fine structure of charged particle spectra among light nuclei, where the Coulomb barrier is low. This fine structure could play an important role, for example, in extracting the beta-decay strength for the parent nuclei and in deriving information about the excited states and their wave functions in the form of spectroscopic factors.

The proton-rich nuclide ${ }^{33} \mathrm{Ar}$ has a beta-decay Q-value of $11.62 \mathrm{MeV}$ [1] and due to the low proton separation energy $2276.5 \pm 0.5 \mathrm{keV}$ in the daughter nuclide ${ }^{33} \mathrm{Cl}$, high-lying states can best be studied by observing beta-delayed protons. This nuclide, with $\mathrm{T}_{z}=-3 / 2$, has been studied extensively by Hardy et al. [2] and Borge et al. [3], who deduced the reduced GT-strength and also by Schardt and Riisager [4], who extracted the GT-strength to the Isobaric Analogue State (IAS) by measuring the broadening in the proton peak caused by the beta-neutrino recoil effect. Based on the measurement of the intensity of the $2230 \mathrm{keV}$

gamma-ray in ${ }^{32} \mathrm{~S}$, a value of $0.8 \%$ could be determined for the total proton branch to this excited state [3]. None of the earlier measurements was able to detect low-energy protons, due to intense beta background and/or the energy threshold of the detector systems. With this in mind, we have restudied the beta-delayed charged-particle and $\gamma$-decay of ${ }^{33} \mathrm{Ar}$ with intense and high quality sources produced at the ISOLDE PSB Facility at CERN. Charged particle decays were measured using a low-energy detector telescope system with a detection threshold for protons of about $200 \mathrm{keV}$ and good energy resolution. The setup was constructed for and used in a study of the mechanism of beta-delayed two-proton decay of the $\mathrm{T}_{z}=-5 / 2$ nucleus, ${ }^{31} \mathrm{Ar}$. In this experiment, the ${ }^{33}$ Ar source was produced and used for energy and efficiency calibrations.

\section{Experimental techniques}

The short-lived Ar ions were produced in spallation reactions in a $\mathrm{CaO}$ target by the 1 $\mathrm{GeV}$ proton beam from the PS-Booster at CERN. The transfer line from the target to the ion source was kept below $1000 \mathrm{~K}$ to ensure high selectivity for a noble gas. Argon ions were produced in a plasma ion source and subsequently accelerated to $60 \mathrm{keV}$ energy and mass separated by the General Purpose Separator of the ISOLDE PSB Facility. The low energy ${ }^{33} \mathrm{Ar}$ ions were implanted into a thin carbon foil, providing an excellent high- 
resolution source for spectroscopic measurements. The collection rate of ${ }^{33} \mathrm{Ar}$ was about 1000 ions/s. The total number of decay events recorded in a 2 hour experiment was about $10^{5}$.

The experimental setup consisted of a conventional silicon $\Delta \mathrm{E}-\mathrm{E}$ telescope, a novel gas-Si combination detector telescope and one annular Si detector for charged particles. For the measurements of beta-delayed $\gamma$-rays, a $70 \%$ germanium detector was used. Lowenergy beta-delayed protons could be identified with the specially designed gas-silicon detector telescope, see refs. $[5,6]$. The gas- $\Delta \mathrm{E}$ detector has an effective thickness of only $70 \mu \mathrm{g} / \mathrm{cm}^{2}$, consisting of a $45 \mu \mathrm{g} / \mathrm{cm}^{2}$ thick polypropylene entrance window and an 8.5 mm thick volume of $C F_{4}$ gas at a pressure of 10 mbar. Three equally spaced tungstenmultiwire electrodes were used to extract the signal produced by the transmitted ion. The thickness of the wire used in the electrodes is $20 \mu \mathrm{m}$ and the total transmission of the grid system is about $94 \%$. The thickness of the E-detector was $300 \mu \mathrm{m}$. The efficiency of this telescope was measured to be $2.5 \%$. Because of the very small energy loss in the $\Delta \mathrm{E}$ transmission detector it was possible to construct a nearly linear energy calibration for the E-detector without adding the energy deposited in the $\Delta$ E-detector, which was used for particle identification only. The known proton energies from ref. [4], with an uncertainty of less than $2 \mathrm{keV}$ and with intensity greater than $2 \%$ of the strongest proton peak were used for energy calibration. The calibration was performed using the energies obtained after subtracting the calculated $\Delta$ E-values. This internal energy calibration was used and was checked with the beta-delayed proton precursor ${ }^{32} \mathrm{Ar}$ which has a known low-energy proton line at $602 \pm 5 \mathrm{keV}[4]$.

\section{Results}

\subsection{Proton spectrum}

The proton spectrum measured over a period of 2 hours using the detector telescope described in Chapter 2 is shown in Fig. 1. This spectrum was generated using the twodimensional gating on the $\Delta \mathrm{E}-\mathrm{E}$ distribution of events. The energy range covered in this spectrum extends from $250 \mathrm{keV}$ up to about $6000 \mathrm{keV}$. At energies above $1 \mathrm{MeV}$ the spectrum is in good agreement with previous works. In addition, a prominent new proton peak at $768 \pm 5 \mathrm{keV}$ is clearly visible. A continuum in the spectrum around $1.5 \mathrm{MeV}$ is due to protons from the very intense $3.17 \mathrm{MeV}$ proton peak suffering energy loss in the tungsten wire electrodes of the $\Delta$ E-detector. Another distortion in the spectrum is observed in the form of the "sum-peak" between 3.3 and $3.4 \mathrm{MeV}$ being due to the simultaneous detection of protons from the IAS and the preceeding beta in the E-detector of the telescope. The new low-energy proton group is proposed to de-excite the $5296 \pm 2 \mathrm{keV}$ level in ${ }^{33} \mathrm{Cl}$. This level was reported by Schardt and Riisager [4] in their high-resolution study performed 
earlier at ISOLDE. The level was observed via the $2927 \pm 2 \mathrm{keV}$ proton transition to the ground state in ${ }^{32} \mathrm{~S}$. The reported energy of this state is in good agreement with the present value of $5297 \pm 3 \mathrm{keV}$ as derived from the energy of the observed $768 \mathrm{keV}$ peak. The assignment is based mainly on the energetics, but also on the width of this peak. The transitions to the excited states are associated with smaller width than those to the ground state, simply due to the kinematic effects as described in ref. [4]. The full width at half maximum measured for the $768 \mathrm{keV}$ line was $23.5 \mathrm{keV}$. This should be compared with the width of $23.2 \mathrm{keV}$ for the known $1322 \mathrm{keV}$ transition to the excited state and the width of $35.6 \mathrm{keV}$ for the $2096 \mathrm{keV}$ transition to the ground state of ${ }^{32} \mathrm{~S}$. The assignment to the beta decay of ${ }^{33} \mathrm{Ar}$ was based on the similarity in half-life of this peak and the strongest proton peak at $3.17 \mathrm{MeV}$ in the spectrum. In addition, there is no other known state in ${ }^{33} \mathrm{Cl}$ that could be associated with the $768 \mathrm{keV}$ proton group, either leading to the ground state or to any of the excited states of ${ }^{32} \mathrm{~S}[8]$. The $768 \mathrm{keV}$ proton decay was not possible to observe in coincidence with the gamma transition to the ground state of ${ }^{32} \mathrm{~S}$ due to low statistics.

\subsection{Partial level scheme}

Based on the previous assignment and the known literature, a partial level and decay scheme of ${ }^{33} \mathrm{Cl}$ was constructed in the neighborhood of the $1 / 2^{+}, T=3 / 2$ IAS at $5544 \mathrm{keV}$. In this scheme, as shown in Fig. 2, all known positive parity states between 4.8 and 6.3 $\mathrm{MeV}$ populated in the beta decay of ${ }^{33} \mathrm{Ar}$ are displayed. The beta-decay branches are given in $\%$ of the decay. They were derived using the $30 \%$ feeding of the IAS as a reference. This branch was calculated according to the procedure of ref. [3] by using the upper limit 0.029 for the Gamow-Teller strength, as given in ref. [4]. This calculation did not include the correction due to the isospin purity of the IAS, which is not known for this state. However, a simple estimate can be made from the sum of isospin mixing amplitudes between the nearby $1 / 2^{+}(\mathrm{T}=1 / 2)$ states as given in ref. [7]. However, the resulting value of $99 \%$ would not influence the values of the beta decay branching ratios given in Fig. 2. The upper limit $0.11 \%$ of the beta-decay branch to the $5738 \mathrm{keV} 1 / 2^{+}$ state was determined from the "sum-free" proton spectrum taken in coincidence with beta particles detected in the opposite Si-detector. This value agrees with an intensity of $0.03(6) \%$ given in ref. [4].

There are two rather special features in the excited states of ${ }^{33} \mathrm{Cl}$. Firstly, the proton decay of the isobaric analogue state (IAS) is isospin forbidden and is possible only via the mixing of the initial and/or the final state. In this case, mainly via the mixing of the initial states. For mixing there is one state $96 \mathrm{keV}$ below and another one $194 \mathrm{keV}$ above the IAS. An interesting similarity between these states and the IAS is their decay predominantly $(=100 \%)$ to the ground state of ${ }^{32} \mathrm{~S}$ by the emission of $l=0$ protons. Secondly, there are two states in the partial level scheme of Fig. 2 which preferably decay to the first excited $2^{+}$-state in ${ }^{32} \mathrm{~S}$. The level at $5870 \mathrm{keV}$ decays $>90 \%$ to the $2^{+}$-state and the level at 
5297 decays to this state with a branching of about $30 \%$ and to the ground state with a branching of about $70 \%$. The spins and parities of these states can be fixed as $(1 / 2,3 / 2)^{+}$ based on their feeding in the allowed beta decay. Judging from their very different decay pattern these states do not seem to be mixing with the IAS, i.e. a spin-parity value of $1 / 2^{+}$is not likely. Moreover, their proton decay to the $2^{+}$-state could be favoured by the angular-momentum barrier penetrability compared with the ground state decay, if the states were assigned $3 / 2^{+}$character. For these two reasons, spin-parity of $3 / 2^{+}$is assigned to all mentioned states decaying to the first $2^{+}$-state in ${ }^{32} \mathrm{~S}$.

Based on the results obtained in this work, a total of six states with proton decay to the first excited state in ${ }^{32} \mathrm{~S}$ could be identified. The summary of all the transition pairs measured in this work is collected in Table 1. A good agreement for the 7471 and 7545 $\mathrm{keV}$ states reported in ref. [4] is observed. The table also includes the calculated barrier penetrabilities and the deduced spectroscopic amplitudes derived in a standard manner similar to the one described in ref. [9]. The barrier penetrabilities were calculated from the regular and irregular Coulomb wave functions with a nuclear radius parameter $\mathrm{r}_{0}=1.3 \mathrm{fm}$, see ref. [2].

\section{Shell Model calculations}

Detailed calculations of the beta strength of ${ }^{33} \mathrm{Ar}$ and of the excited states of ${ }^{33} \mathrm{Cl}$ were performed by Brown and Wildenthal [10]. They reproduce well the gross features of the decay, but the overall strength is found to behave anomalously in comparison with other $s d$-shell nuclei. In fact, the amount of strength predicted inside the energy window of the beta decay is nearly equal to what is experimentally measured, while in most other cases the predicted strength overshoots the measured one by a factor $1 /\left(.77^{2}\right)$ [11]. It has become clear that this is a particular default behaviour of the interaction used in ref. [10], named $\mathrm{W}$-interaction, that, close to $\mathrm{Z}=20$ and for proton rich nuclei, shifts the Gamow-Teller resonance to higher energies. This effect was discussed by Brown in his analysis of the ${ }^{37} \mathrm{Ca}$ decay [12]. Instead of the W-interaction, we can use the Chung-Wildenthal one for the upper part of the sd-shell (denoted CWH). This interaction gives results similar to the W ones for nuclei closer to the stability, but when used far off stability the "anomalous" behaviour of the Gamow-Teller strength disappears and a standard $\left(.77^{2}\right)$ quenching is needed to bring theory and experiment into agreement. Actually, in the case of ${ }^{33} \mathrm{Ar}$, the total Gamow-Teller strength within the beta-decay energy window, in units of the $3(\mathrm{~N}-\mathrm{Z})$ sum rule, is equal to 7.22 for the CWH-interaction while it is only 4.33 for the W-interaction.

We have made full $s d$-shell calculations with the two interactions mentioned above using the shell-model code ANTOINE [13]. In order to have a measure of the effect of the structure of mother and daughter states in the ${ }^{33} \mathrm{Cl}$ proton emission probability, we have 
computed the spectroscopic amplitudes

$$
\left|<{ }^{33} \mathrm{Cl}\left(J_{f}\right)\right| a_{j}^{\dagger}\left|{ }^{32} \mathrm{~S}\left(3 / 2^{+}\right)_{n}>\right|^{2}
$$

for the $\mathrm{j}$-orbits $1 d_{3 / 2}, 2 s_{1 / 2}$ and $1 d_{5 / 2}$, to the ground state $\left(0^{+}\right)$and to the first excited state $\left(2^{+}\right)$of ${ }^{32} \mathrm{~S}$.

The results are summarized in Table 2. It is quite clear that, for the states we are interested in, the spectroscopic amplitudes of the transitions to the $2^{+}$state are larger. In order to compare with the experimentally extracted "enhancements", we have averaged the results for the three orbits, then we have obtained, for every state, the ratios between the branches to the $0^{+}$and to the $2^{+}$states. These values are listed in the lower part of Table 2 . The results for both interactions are compatible with the experimental numbers but the CWH values are definitely higher. These results imply that the decay to the excited states in the daughter nuclei can be an important process and should be taken into account even for small Q-value decays, for example, when deducing the beta-strength distribution from the experimental particle spectra. On a more detailed level, two experimentally observed states at 5297 and $5870 \mathrm{keV}$ having strongest relative branching to the $2^{+}$state are also predicted by the shell model. The theoretical values for the energies of these states are 5.08 and $5.31 \mathrm{MeV}$ with the W-interaction and 5.21 and $5.61 \mathrm{MeV}$ with the CWH-interaction. Further justification for this assignment is obtained from the feeding of the beta decay of

${ }^{33}$ Ar. The calculated values using the W-interaction [10] give the branching ratio of about $0.3 \%$ for both states, which compares well with the values shown in Fig. 2.

\section{Conclusions}

The experimental technique presented in this work provides a highly sensitive and highresolution probe for studying fine structure of the beta-delayed particle emission. Using this technique and the thin and nearly point-like sources provided by ISOLDE, fine structure of the beta-delayed proton decay of ${ }^{33} \mathrm{Ar}$ could be studied over a wide energy range. Several strong proton transitions to the first excited $2^{+}$-state in ${ }^{32} \mathrm{~S}$ were observed. This observation allowed exact testing of the shell model calculations for the spectroscopic amplitudes of the high-lying excited states. A good qualitative agreement was observed for the ratios of the spectroscopic amplitudes of the transitions leading to the first excited state and the ground state in ${ }^{32} \mathrm{~S}$ with both interactions used in the calculation. At the same time, rather large difference between two interactions is observed in the calculated beta decay strengths calling for the adjustment of the interaction used and/or for enlargening the configuration space of the calculation. 


\section{Acknowledgements}

This work has been financially supported by the Academy of Finland and the CICYT (Spain, under contract AEN94-0833-C02-02) The authors would like to thank Dr. K. Riisager and P. Hornshoj for their valuable comments on the manuscript.

\section{References}

[1] G. Audi and A.H. Wapstra, Nucl. Phys. A565 (1993) 1

[2] J.C. Hardy, J. Esterl, R. Sextro and J. Cerny, Phys. Rev. C3 (1973) 700

[3] M.J.G. Borge, et al., Physica Scripta, 36 (1987) 218

[4] D. Schardt and K. Riisager, Z. Phys. A345 (1993) 265

[5] D. M. Moltz, J. D. Robertson, J. C. Batchelder and J. Cerny, Nucl. Instr. Meth. A349 (1994) 210

[6] A. Honkanen, et al., to be published.

[7] W.E. Ormand and B.A. Brown, Phys. Lett. B174 (1986) 128

[8] P.M. Endt, Nucl. Phys. A521 (1990) 1

[9] B.A. Brown, Phys. Rev. Lett. 65 (1990) 2753

[10] B.A. Brown and B. H. Wildenthal, At. Data Nucl. Data Tables 33 (1985) 347

[11] B. Jonson and G. Nyman, in Nuclear Decay Modes, IAP, Bucharest, Ed. D.N. Poenaru. p. 102

[12] B.A. Brown, Phys. Rev. Lett. 69 (1992) 1034

[13] E. Caurier, Shell Model code ANTOINE, Strasbourg 1989. 


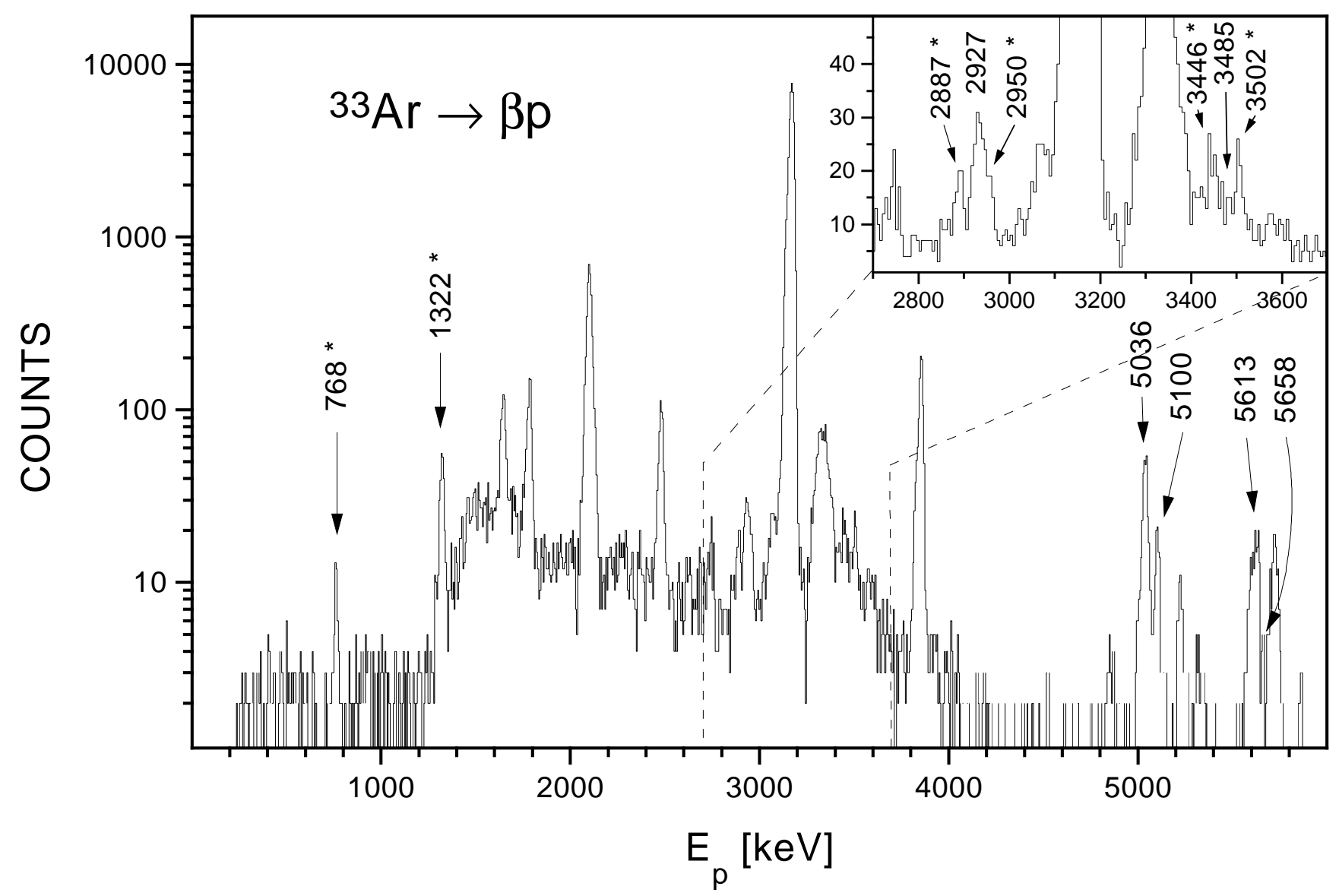

Figure 1: Beta-delayed proton spectrum of ${ }^{33} \mathrm{Ar}$ as observed using the low-energy detector telescope. Only those transition pairs observed to decay both to the ground state and the first excited $2^{+}$state in ${ }^{32} \mathrm{~S}$ are shown. The asterisk denotes the transition to the excited state. 


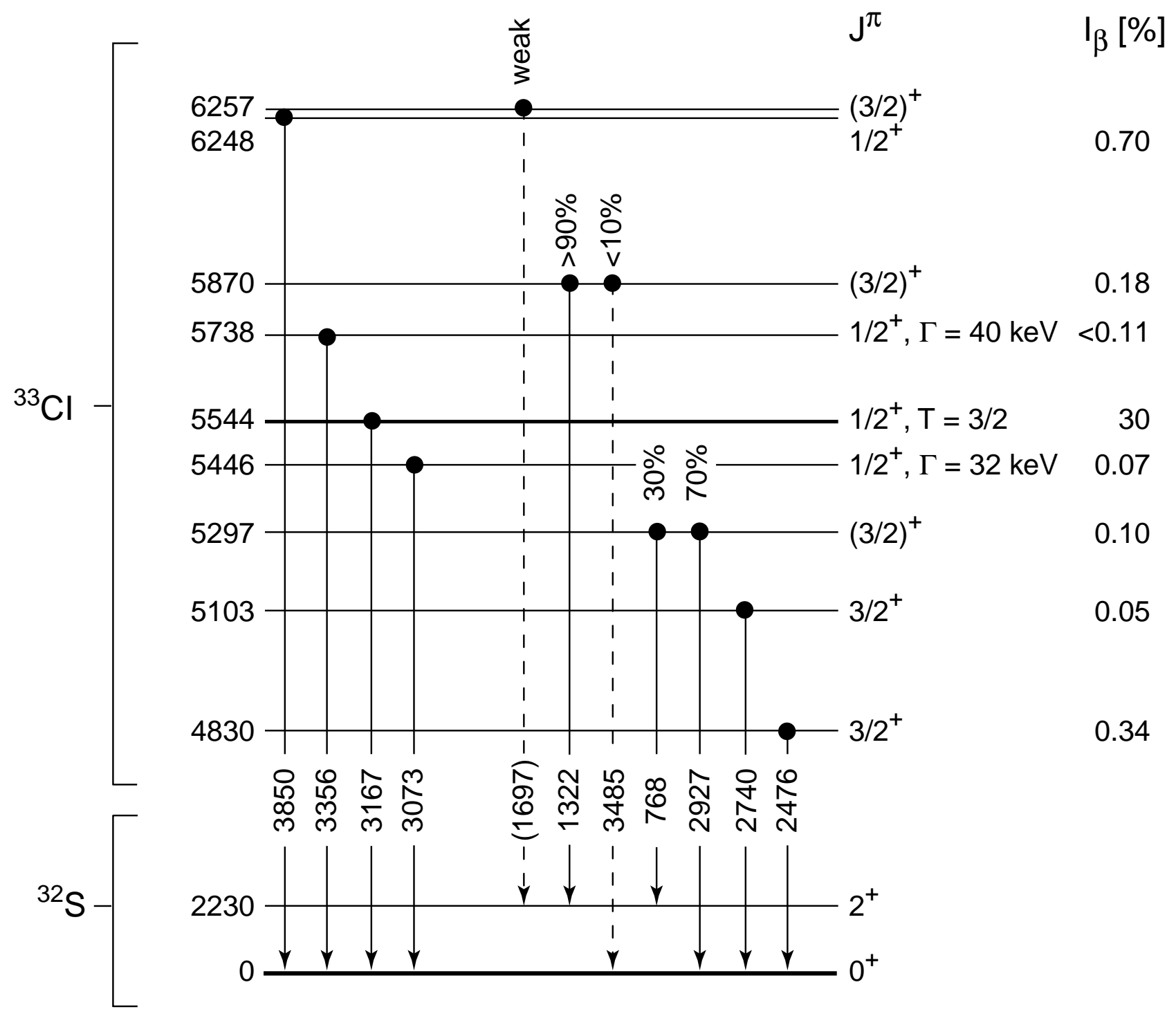

Figure 2: Partial level scheme of ${ }^{33} \mathrm{Cl}$. For the more complete decay and level schemes, see refs. $[2,3,4]$. 
Table 1: Summary of the excited states in ${ }^{33} \mathrm{Cl}$, decaying by proton emission to the $0^{+}$ ground state and the first excited $2^{+}$state in ${ }^{32} \mathrm{~S}$. The spin-parity of $3 / 2^{+}$is assumed in the penetrability calculation, requiring $l=0$ and $l=2$ for the decay to the excited state and the ground state, respectively. The last column gives the ratio of the spectroscopic amplitudes for the decays to the excited $2^{+}$and the $0^{+}$ground state in ${ }^{32} \mathrm{~S}$.

\begin{tabular}{|c|c|c|c|c|c|}
\hline $\mathrm{E}_{p}[\mathrm{keV}]$ & $\mathrm{E}_{x}[\mathrm{keV}]$ & $\begin{array}{l}\mathrm{E}_{x}[\mathrm{keV}] \\
\text { (adopted) }\end{array}$ & $\begin{array}{l}\text { Intensity } \\
\text { (counts) }\end{array}$ & Penetrability & $\Theta\left(2^{+}\right) / \Theta\left(0^{+}\right)$ \\
\hline $768 \pm 5$ & $5299 \pm 5$ & \multirow[t]{2}{*}{$5297 \pm 4$} & $39 \pm 9$ & $1.0 \cdot 10^{-3}$ & \multirow[t]{2}{*}{$6.6_{-1.3}^{+1.6}$} \\
\hline $2927 \pm 4$ & $5296 \pm 4$ & & $93 \pm 19$ & $1.1 \cdot 10^{-1}$ & \\
\hline $1322 \pm 3$ & $5870 \pm 3$ & \multirow[t]{2}{*}{$5870 \pm 3$} & $251 \pm 18$ & $3.5 \cdot 10^{-2}$ & \multirow[t]{2}{*}{$>8$} \\
\hline $3485 \pm 15$ & $5871 \pm 15$ & & $<20$ & 0.21 & \\
\hline $2887 \pm 10$ & $7485 \pm 10$ & \multirow[t]{2}{*}{$7471 \pm 4$} & $48 \pm 18$ & 0.62 & \multirow[t]{2}{*}{$0.41 \pm 0.10$} \\
\hline $5036 \pm 4$ & $7471 \pm 4$ & & $292 \pm 19$ & 0.63 & \\
\hline $2950 \pm 5$ & $7550 \pm 5$ & \multirow[t]{2}{*}{$7545 \pm 5$} & $48 \pm 18$ & 0.65 & \multirow[t]{2}{*}{$0.68 \pm 0.17$} \\
\hline $5100 \pm 4$ & $7539 \pm 4$ & & $104 \pm 12$ & 0.65 & \\
\hline $3446 \pm 7$ & $8061 \pm 7$ & \multirow[t]{2}{*}{$8066 \pm 6$} & $47 \pm 15$ & 0.89 & \multirow[t]{2}{*}{$0.52 \pm 0.11$} \\
\hline 5613 & $8066 \pm 6$ & & $160 \pm 14$ & 0.81 & \\
\hline $3502 \pm 7$ & $8119 \pm 7$ & \multirow[t]{2}{*}{$8119 \pm 7$} & $48 \pm 15$ & 0.91 & \multirow[t]{2}{*}{$>3$} \\
\hline $5658 \pm 10$ & $8112 \pm 10$ & & $<6$ & 1 & \\
\hline
\end{tabular}


Table 2: Spectroscopic amplitudes corresponding to the transitions between the $0^{+}$ground state and the first excited $2^{+}$state in ${ }^{32} \mathrm{~S}$ and the $3 / 2^{+}$states in ${ }^{33} \mathrm{Cl}$. The calculation is performed with both the universal sd-shell (W) and the Chung-Wildenthal (CWH) interaction. Only the $3 / 2^{+}$states of relevance for this work, i.e. between 4 and $6.5 \mathrm{MeV}$ excitation are shown. The lower part of the table shows the average ratio of the three amplitudes.

\begin{tabular}{|c|c|c|c|c|c|}
\hline Interaction & ${ }^{32} \mathrm{~S}$ & $\begin{array}{l}\mathrm{E}_{x} \text { in }{ }^{33} \mathrm{Cl} \\
{[\mathrm{MeV}]}\end{array}$ & $d_{3 / 2}$ & $s_{1 / 2}$ & $d_{5 / 2} / /$ \\
\hline \multirow[t]{4}{*}{ W } & $0^{+}$ & 4.81 & 0.008 & 0.0 & 0.0 \\
\hline & & 5.08 & 0.009 & 0.0 & 0.0 \\
\hline & & 5.31 & 0.005 & 0.0 & 0.0 \\
\hline & & 6.55 & 0.009 & 0.0 & 0.0 \\
\hline \multirow[t]{4}{*}{ CWH } & $0^{+}$ & 4.84 & 0.006 & 0.0 & 0.0 \\
\hline & & 5.21 & 0.008 & 0.0 & 0.0 \\
\hline & & 5.61 & 0.000 & 0.0 & 0.0 \\
\hline & & 6.34 & 0.012 & 0.0 & 0.0 \\
\hline \multirow[t]{4}{*}{ W } & $2^{+}$ & 4.81 & 0.005 & 0.002 & 0.000 \\
\hline & & 5.08 & 0.007 & 0.037 & 0.001 \\
\hline & & 5.31 & 0.029 & 0.006 & 0.003 \\
\hline & & 6.55 & 0.032 & 0.000 & 0.006 \\
\hline \multirow[t]{5}{*}{$\mathrm{CWH}$} & $2^{+}$ & 4.84 & 0.013 & 0.004 & 0.016 \\
\hline & & 5.21 & 0.036 & 0.066 & 0.000 \\
\hline & & 5.61 & 0.019 & 0.015 & 0.001 \\
\hline & & 6.34 & 0.030 & 0.006 & 0.014 \\
\hline & & & Ratio & & \\
\hline \multirow[t]{4}{*}{$\mathrm{W}$} & $2^{+} / 0^{+}$ & 4.81 & 0.9 & & \\
\hline & & 5.08 & 5.0 & & \\
\hline & & 5.31 & 7.6 & & \\
\hline & & 6.55 & 4.2 & & \\
\hline \multirow[t]{4}{*}{ CWH } & $2^{+} / 0^{+}$ & 4.84 & 5.5 & & \\
\hline & & 5.21 & 12.8 & & \\
\hline & & 5.61 & $>35$ & & \\
\hline & & 6.34 & 4.2 & & \\
\hline
\end{tabular}

